\title{
Efecto de la heterogeneidad espacial y estacional del suelo sobre la abundancia de esporas de hongos micorrizógenos arbusculares en el valle semiárido de Tehuacán-Cuicatlán, México
}

\author{
Sara Lucía Camargo-Ricalde \& Manuel Esperón-Rodríguez \\ Universidad Autónoma Metropolitana-Iztapalapa, División de Ciencias Biológicas y de la Salud, Departamento de Biología; Apdo. \\ Postal 55-535, 09340 México, D. F. Fax: (52)(55) 58-04-46-88; slcr@xanum.uam.mx / orcacomefoca@ yahoo.com.mx
}

Recibido 22-IV-2005. Corregido 29-VI-2005. Aceptado 31-VIII-2005.

\begin{abstract}
Effect of the spatial and seasonal soil heterogeneity over arbuscular mycorrhizal fungal spore abundance in the semi-arid valley of Tehuacán-Cuicatlán, Mexico. Recent studies have shown that some species of Mimosa (Leguminosae-Mimosoideae) create resource islands (RI), rich in soil organic matter and nutrients, as well as in arbuscular mycorrhyzal fungal (AMF) spores, in the semi-arid Valley of TehuacánCuicatlán. The relevance of this fact is that arid and semi-arid regions are characterized by low fertility soils and scarce precipitation, limiting plant species growth and development; this explains why the presence of AM fungi may be advantageous for mycorrhizal desert plants. Fluctuations in AMF spore numbers could be related to environmental, seasonal and soil factors which affect AMF sporulation, in addition to the life history of the host plant. The aim of this study was to asses the impact of spatial (resource islands vs open areas, OA) and seasonal (wet season vs start of dry season vs dry season) soil heterogeneity in the distribution and abundance of AMF spores in four different study sites within the Valley. We registered AMF spores in the 120 soil samples examined. Significant differences in the number of AMF spores were reported in the soil below the canopy of Mimosa species (RI) comparing with OA (RI > OA), and between Mimosa RI themselves when comparing along a soil gradient within the RI (soil near the trunk > soil below the middle of the canopy > soil in the margin of the canopy > OA); however, there were no significant differences between the soil closest to the trunk $v$ s middle, and margin $v s$ OA. Finally, more spores were reported in the soil collected during the wet season than during the dry season (wet > start of dry > dry). Therefore, the distribution of AMF spores is affected by spatial and seasonal soil heterogeneity. This study points out the relevance of Mimosa RI as AMF spore reservoirs and the potential importance of AM fungi for plant species survivorship and establishment in semi-arid regions. AM fungi have recently been recognized as an important factor determining plant species diversity in arid and temperate ecosystems. Rev. Biol. Trop. 53(3-4): 339-352. Epub 2005 Oct 3.
\end{abstract}

Key words: arbuscular mycorrhizal fungal (AMF) spores, soil seasonal heterogeneity, soil spatial heterogeneity, Mimosa, resource islands, semi-arid.

En una primera aproximación, CamargoRicalde et al. (2002) demostraron que algunas especies de Mimosa (LeguminosaeMimosoideae) forman islas de recursos (IR) en el Valle semiárido de Tehuacán-Cuicatlán, Puebla, ricas no sólo en materia orgánica y nutrientes del suelo (NT, $\mathrm{P}, \mathrm{Ca}, \mathrm{Mg}$ ), sino en esporas de hongos micorrizógenos arbusculares (HMA) (Camargo-Ricalde y Dhillion 2003). La relevancia de este hecho radica en que las zonas áridas y semiáridas se caracterizan, principalmente, por la baja fertilidad del suelo y la escasa precipitación, limitando el crecimiento y desarrollo de las especies vegetales, por lo que la presencia de HMA puede ser una ventaja para las plantas de zonas áridas y semiáridas que forman micorrizas (Dhillion y Zak 1993, Tarafdar y Praveen-Kumar 1996). Para las zonas áridas y semiáridas, se ha determinado la importancia de las micorrizas en el 
establecimiento y en el mantenimiento de la diversidad vegetal, así como en el funcionamiento y la restauración de estos ecosistemas (Allen y Allen 1986, Whitford 1996, Hartnett y Wilson 1999, Koide et al. 2000). De manera general, se ha sugerido que en las comunidades terrestres, la diversidad de especies vegetales está determinada por la presencia de HMA en el suelo (Turner y Friese 1998, Van der Heijden et al. 1998a, 1998b, Hartnett y Wilson 1999); asimismo, se tienen evidencias de que las especies vegetales y las interacciones que éstas presentan con los microorganismos del suelo se modifican en presencia de estos hongos (Newsham et al. 1995, Streitwolf-Engel et al. 1997, Titus y del Moral 1998a, 1998b).

Las comunidades de HMA presentan diferencias en densidad relativa, producción de esporas y funciones correlacionadas con el ambiente; por ejemplo, al comparar entre la estación de lluvias y la estación de sequía, así como con la especificidad del hospedero y la presencia de otras especies de hongos al mostrar interacciones de competencia intra- e interespecífica (Titus y del Moral 1998a, 1998b, Turner y Friese 1998).

Existen grandes variaciones en las estimaciones resultantes de algunos estudios enfocados a determinar el número de esporas de HMA en el suelo de ecosistemas áridos y semiáridos (Mosse y Bowen 1968, Dhillion y Anderson 1993, Dhillion et al. 1994, Jacobson 1997, Camargo-Ricalde y Dhillion 2003). Al respecto, se sugiere que estas diferencias numéricas pueden estar ligadas con factores ambientales y edáficos; así como a factores estacionales de la esporulación de estos hongos en relación con la especie vegetal hospedera. De ahí la importancia de continuar realizando estudios sobre el ciclo de vida de los HMA a través de la dinámica poblacional de los HMA, principalmente de sus esporas.

El objetivo de este estudio fue el de evaluar el impacto de la heterogeneidad espacial (islas de recursos formadas por especies de Mimosa vs áreas abiertas) y estacional (estación de lluvias, inicio de la estación de sequía y sequía) del suelo en la distribución y abundancia de esporas de HMA, en cuatro sitios de estudio en el Valle de Tehuacán-Cuicatlán, México.

\section{MATERIALES Y MÉTODOS}

Sitios de estudio: El Valle de TehuacánCuicatlán (parte del Valle fue decretado Reserva de la Biósfera en 1998) se localiza entre los $17^{\circ} 20^{\prime}-18^{\circ} 53^{\prime}$ 'N y $96^{\circ} 55^{\prime}-97^{\circ} 44^{\prime} \mathrm{W}$, cubriendo un área aproximada de $10000 \mathrm{~km}^{2}$. El Valle abarca parte de los estados de Puebla y Oaxaca, México. Las altitudes oscilan entre los 500 y $3200 \mathrm{msnm}$. El promedio anual de precipitación es 400-600 mm con régimen de lluvias de verano y la temperatura media anual es de $20^{\circ} \mathrm{C}$. Los suelos son rocosos y poco profundos, bien drenados y principalmente derivados de rocas sedimentarias y metamórficas (Zavala-Hurtado y Hernández-Cárdenas 1998).

Especies de Mimosa seleccionadas: Se seleccionaron cuatro sitios de estudio (S) con base en la presencia de las especies de Mimosa en estudio (endémicas de México*, endémicas del Valle**). Los sitios S1, S2 y S4 se ubican en el matorral xerófilo y el sitio $\mathrm{S} 3$ en restos del bosque tropical caducifolio; ambos tipos de vegetación están perturbados y el primero está categorizado como amenazado (ZavalaHurtado y Hernández-Cárdenas 1998, Camargo Ricalde et al. 2002).

Sitio 1 (S1): $7 \mathrm{~km}$ al NW de Azumbilla, municipio de Chapulco; 18 $41^{\prime} 31^{\prime \prime} \mathrm{N}$, 97²4'01.3" W; 2232 msnm, Mimosa lacerata Rose*.

Sitio 2 (S2): 1 km al S de Coxcatlán, municipio de Coxcatlán; 1853'23.7” N, 9709'03.3”'W; 1140 msnm; Mimosa luisana Brandegee** y Mimosa polyantha Benth*.

Sitio 3 (S3): $4 \mathrm{~km}$ al E-SE de Atexcoco, municipio de Caltepec; $18^{\circ} 12^{\prime} 0.46^{\prime \prime} \mathrm{N}$, 97³1'28.6" W; 2050 msnm; Mimosa texana (A. Gray) Small var. filipes (Britton y Rose) Barneby**.

Sitio 4(S4): 7 km al NW de Los Reyes Metzontla, municipio de Caltepec; $18^{\circ} 16^{\prime} 29.4^{\prime \prime} \mathrm{N}$, 
97³0'12.9” W; 1670 msnm; Mimosa luisana Brandegee*.

Recolecta de suelo: Las recolectas de suelo se llevaron a cabo en dos islas de recursos formadas por cada especie de Mimosa por cada sitio y en dos áreas abiertas (AA) equivalentes en área para cada caso, comparando tres estaciones: 1) Lluvias (julio, 2003); 2) Inicio de sequía (noviembre, 2003) y 3) Sequía (marzo, 2004). Se recolectaron muestras de ca. un kilogramo de suelo entre $0-15 \mathrm{~cm}$ de profundidad: 1) Lo más cercano posible al tronco de Mimosa (tronco), 2) A la mitad de la copa de la planta (medio), 3) En el margen externo de la copa (margen) y 4) En un área abierta (AA).

Las muestras de suelo se recolectaron en bolsas de polietileno negro y se transportaron al laboratorio. Se recolectaron 120 muestras de suelo en total y se analizaron tres submuestras por muestra de suelo para la obtención de esporas de HMA de acuerdo con el método de Gerdemann y Nicolson (1963) (decantación de alícuotas en $100 \mathrm{~g}$ de suelo seco) usando el método de decantación y tamizado húmedo (tamices de 44, 105 y $200 \mu \mathrm{m})$. Se examinaron las alícuotas y se reportó el número de esporas de HMA utilizando un microscopio estereoscópico. Los ejemplares botánicos de referencia se encuentran depositados en el herbario UAMIZ y los viales con las esporas de HMA se encuentran en el laboratorio de Biosistemática de Leguminosas, Depto. de Biología, de la UAM-Iztapalapa.

Asimismo, se relacionó el número de esporas de HMA del suelo con la temperatura ambiental y la precipitación de cada sitio. Los promedios de temperatura y precipitación mensual se obtuvieron con los datos promedio de los meses de recolecta (marzo, julio y noviembre) y de los dos meses anteriores: lluvias (mayo: 39.8-69.2 mm, 21.1-27.5 ${ }^{\circ} \mathrm{C}$; junio: 90.3-96.9 mm, 20-25.6 ${ }^{\circ} \mathrm{C}$; julio: 44.7-75.5 mm, 18.8-24. $6^{\circ} \mathrm{C}$ ), inicio sequía (septiembre: 86.3 $118.3,19-24^{\circ} \mathrm{C}$; octubre: $28.7-36.1 \mathrm{~mm}, 18-$ $23.6^{\circ} \mathrm{C}$; noviembre: $4-6.8 \mathrm{~mm}, 16.5-22.1^{\circ} \mathrm{C}$ ) y sequía (enero: $2.1-11.7 \mathrm{~mm}, 15.1-20.1^{\circ} \mathrm{C}$; febrero: $2.3-5.7 \mathrm{~mm}, 16.4-21.6^{\circ} \mathrm{C}$; marzo: $3.6-$ $\left.16 \mathrm{~mm}, 18.6-24.5^{\circ} \mathrm{C}\right)$. Los datos de temperatura y precipitación se obtuvieron de las estaciones climatológicas correspondientes a cada uno de los sitios de estudio. Los valores son el promedio registrado para las estaciones de Tehuacán: 16 años (S1 y S4), Caltepec: 26 años (S3) y Coxcatlán: 35 años (S2).

Análisis estadístico: Se utilizó la prueba no paramétrica de Kruskal-Wallis $(p<0.05)$ y las medias se compararon con la prueba de $\mathrm{X}^{2}$ $(\alpha=0.05)$ para determinar si había diferencias significativas en el número de esporas de HMA entre el suelo bajo la copa de las especies de Mimosa (IR: tronco, medio y margen) y en el de las AA, durante las tres estaciones analizadas. La prueba no paramétrica de $t$ de Student $(\alpha=0.05)$ se utilizó para determinar si había diferencias significativas en el número de esporas de HMA del suelo entre las tres estaciones analizadas (lluvia, inicio de sequía y sequía) y entre los sitios de estudio.

\section{RESULTADOS}

Se registraron esporas de hongos micorrizógenos arbusculares (HMA) en todas las muestras de suelo analizadas (Fig. 1, Cuadro 1). Con base en la clave de identificación elaborada por Schenck y Perez (1990), los géneros dominantes registrados, hasta ahora, son Acaulospora y Glomus; otras esporas están todavía en proceso de identificación.

No hay diferencia significativa al comparar el número total de esporas de HMA registrado entre las IR en los cuatro sitios de estudio, ni entre el número total de esporas de HMA y el número total de esporas registradas en las AA por sitio.

De manera particular, al comparar entre estaciones, se reportó un mayor número de esporas durante la estación de lluvias (lluvias $>$ inicio de sequía > sequía); de igual forma, el número de esporas registradas en el suelo de las IR formadas por Mimosa fue mayor que el del suelo de las AA. Se obtuvieron diferencias significativas al comparar entre el número de esporas del suelo cercano al tronco y el suelo 


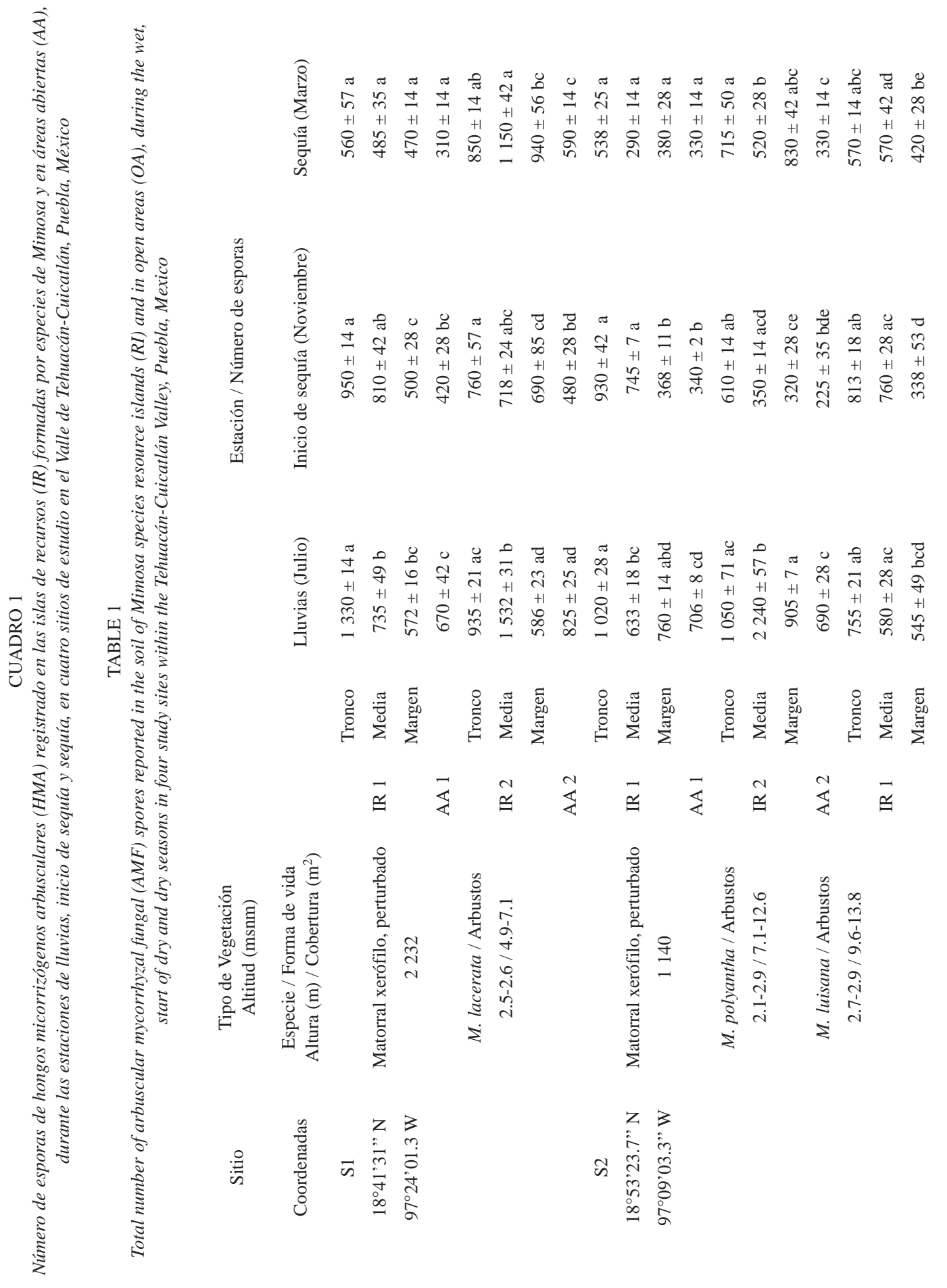




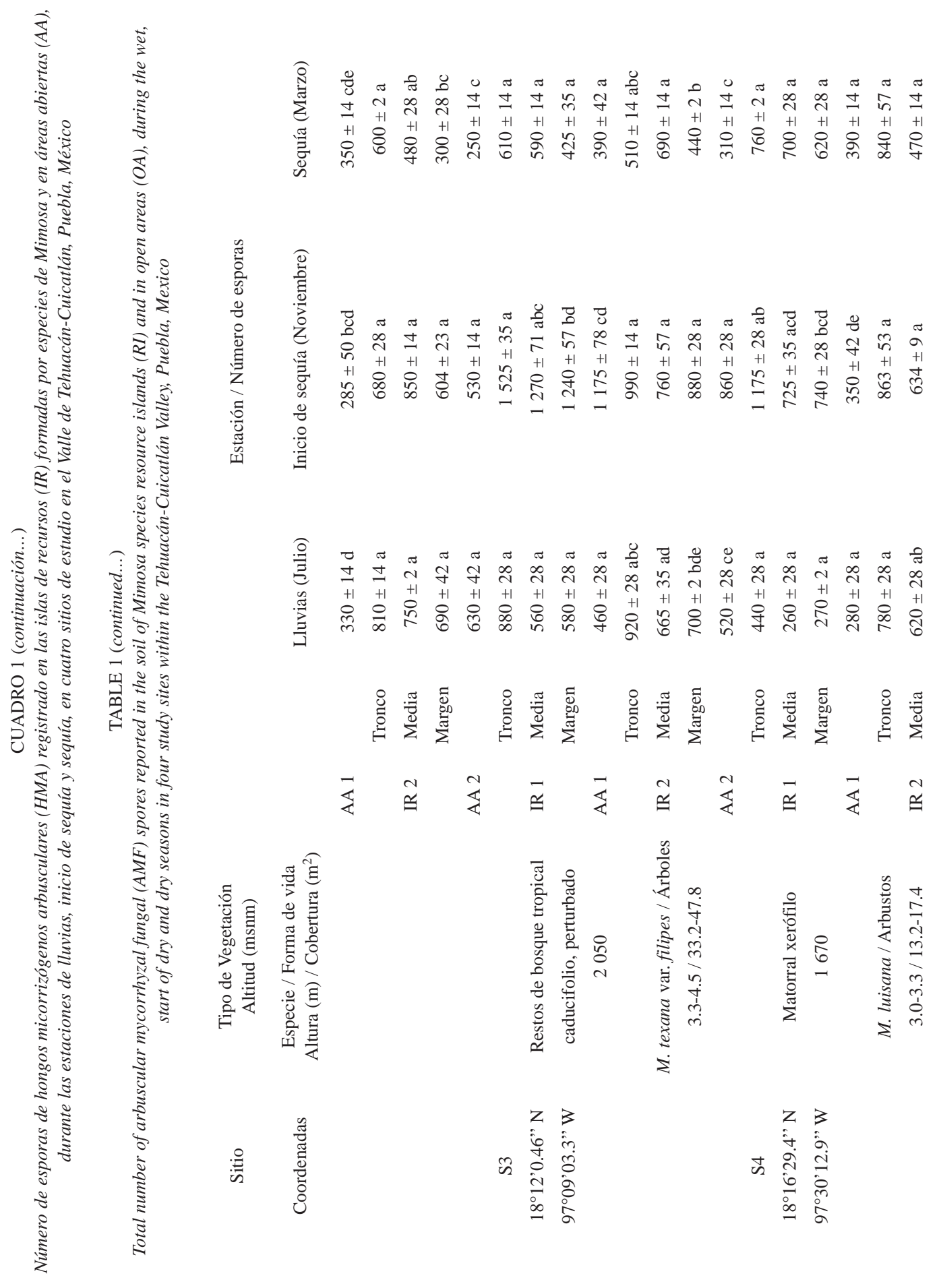




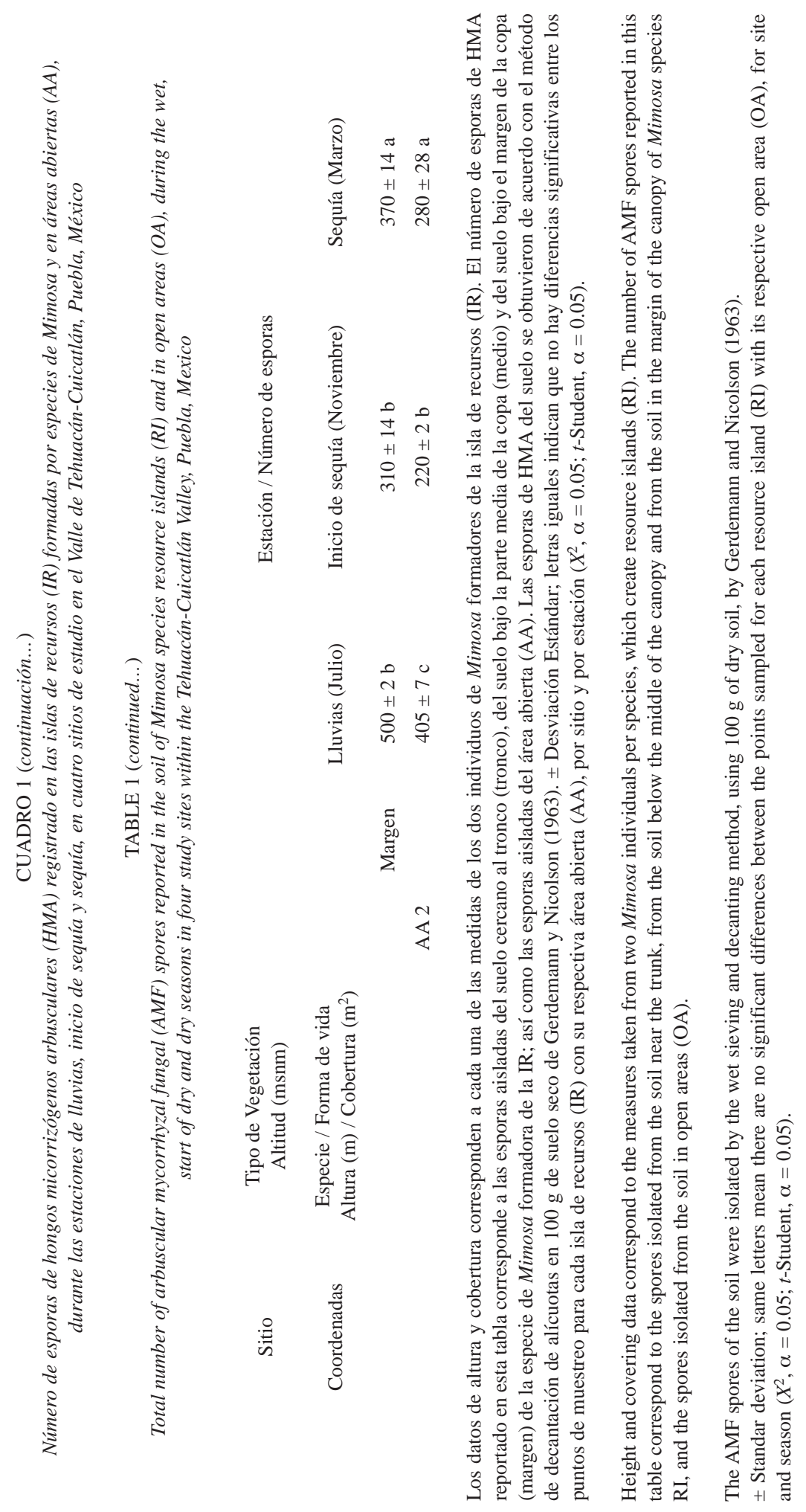



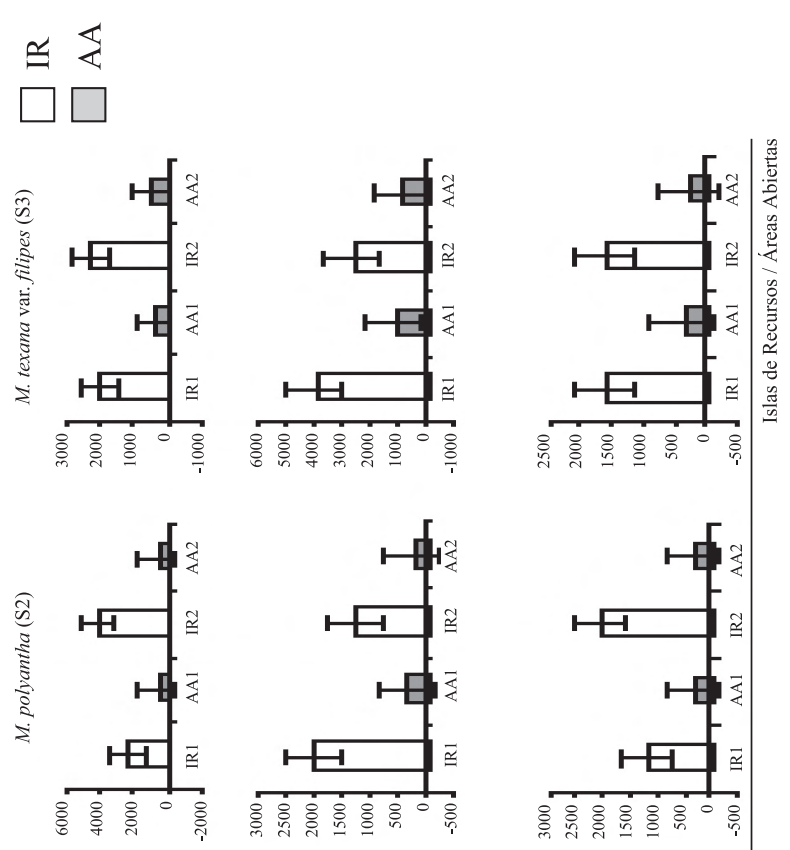

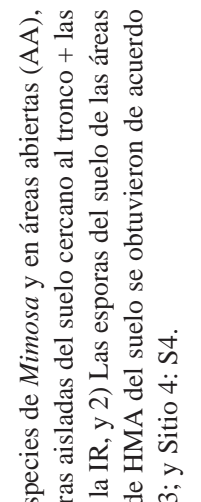

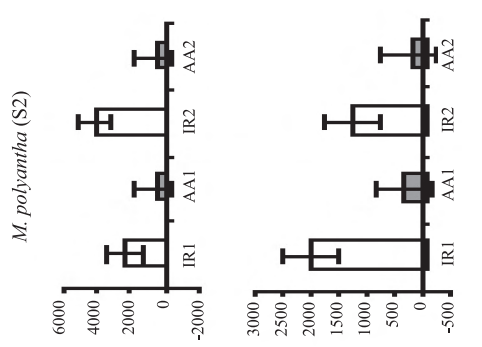

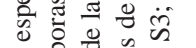

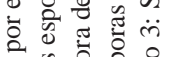

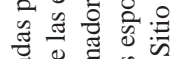

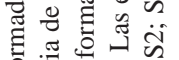

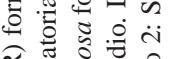

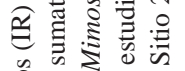

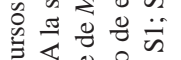

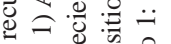

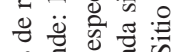
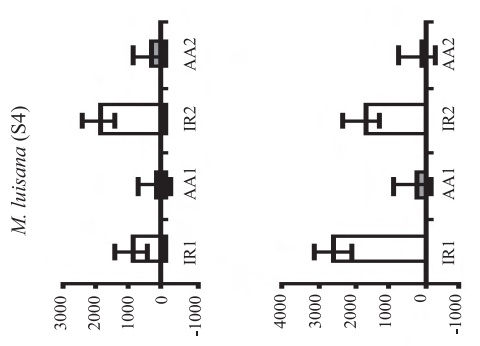

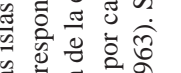

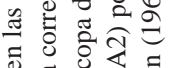

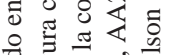

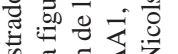

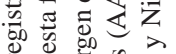

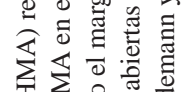

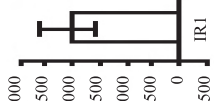
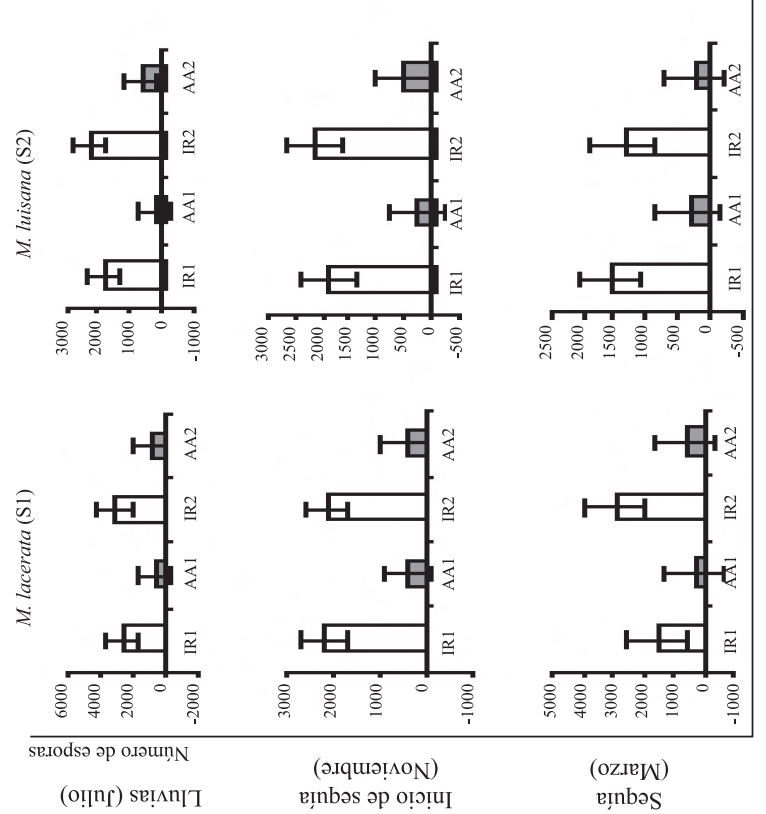

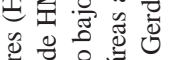

氙

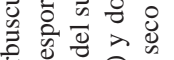

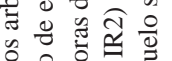

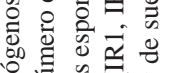

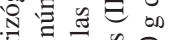

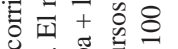

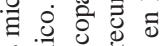

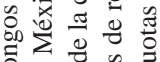

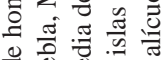

\% 월

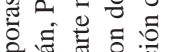

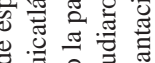

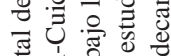

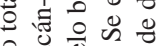

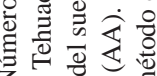

乙. o :

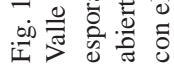

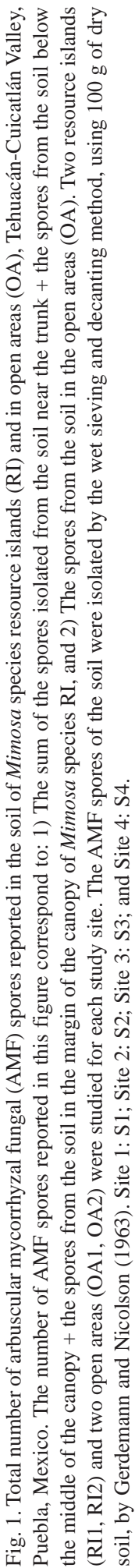


bajo el margen de la copa (tronco $\neq$ margen), y entre el suelo cercano al tronco y el suelo de las AA (tronco $\neq \mathrm{AA}$ ); así como entre el suelo de la parte media de la copa y el suelo del margen de la copa (medio $\neq$ margen), y la parte media de la copa y el suelo de las AA (medio $\neq \mathrm{AA}$ ). Sin embargo, no hubo diferencia significativa al comparar entre el suelo cercano al tronco y el suelo de la parte media de la copa (tronco = medio), y el suelo del margen de la copa y el suelo de las AA (margen = AA). Es importante señalar que no se encontraron diferencias significativas al comparar entre la IR-1 y la IR-2 para cada especie de Mimosa, y entre la AA-1 y la AA-2, para cada uno de los sitios.

Al comparar únicamente entre las especies de Mimosa y las estaciones del año, las diferencias estadísticamente significativas fueron casos muy puntuales:

1) Para M. texana var. filipes (S3): $\neq$ M. lacerata (S1) en inicio de sequía y sequía, $\neq$ $M$. luisana (S2) en inicio de sequía, $\neq=M$. luisana (S4) en lluvias e inicio de sequía, $\neq M$. polyantha $(\mathrm{S} 2)$ en inicio de sequía.

2) Para M. polyantha (S2): $\neq$ M. lacerata (S1) en inicio de sequía, $\neq$ M. luisana (S4) en lluvias.

3) Para M. luisana (S4): $\neq M$. lacerata (S1) en lluvias, $\neq M$. luisana (S2) en lluvias.

4) Para M. luisana (S2): $\neq$ M. lacerata (S1) en sequía.

Además, también existen diferencias significativas muy particulares entre los puntos de muestreo del suelo de las IR (tronco, medio y margen) y el de las AA; las diferencias corresponden a un punto de muestreo específico en una determinada estación del año (Cuadro 2). Asimismo, al comparar entre los puntos de muestreo de las especies de Mimosa y el de las AA, con relación a las estaciones del año, se encontraron diferencias significativas muy específicas (Cuadro 3).

Al relacionar el número de esporas de HMA del suelo con los datos de temperatura ambiental y precipitación media mensual registrados por tres estaciones meteorológicas 


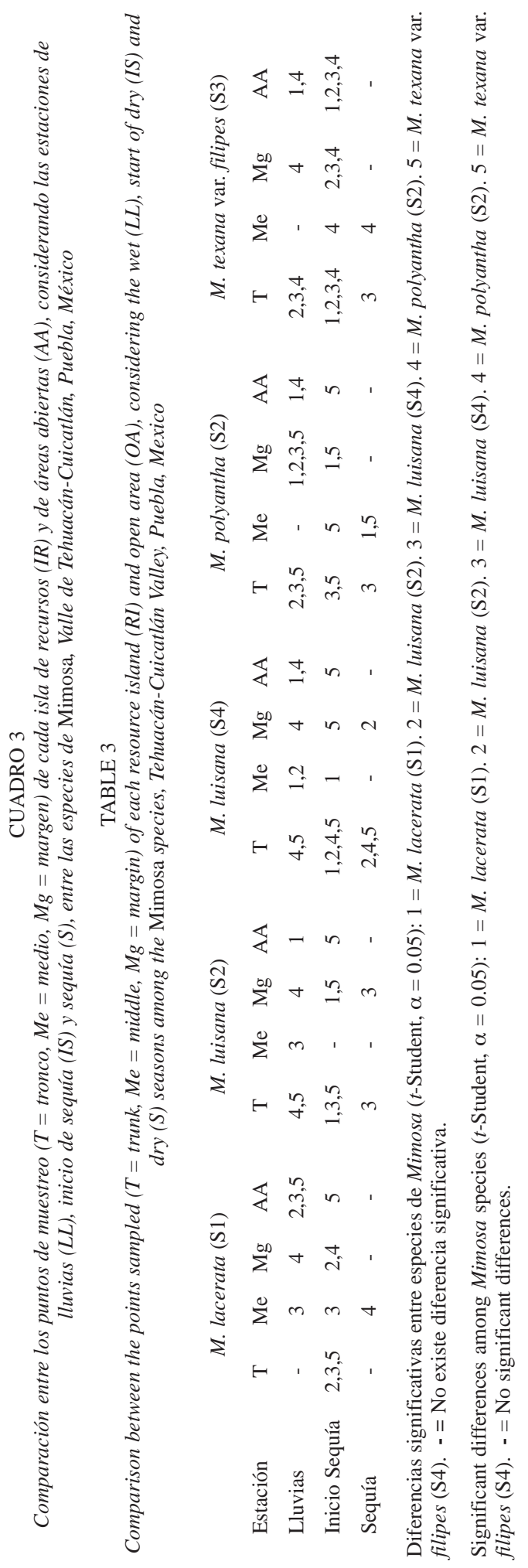

cercanas a los sitios de estudio, se observó que durante la estación de lluvias se presenta el mayor número de esporas de HMA (Fig. 2). En contraste, durante la estación con menor precipitación (inicio de sequía y sequía) se registró el menor número de esporas de HMA (lluvias $>$ inicio de sequía > sequía).

\section{DISCUSIÓN}

Nuestros resultados son coherentes con los obtenidos por Camargo-Ricalde et al. (2002) y Camargo-Ricalde y Dhillion (2003), en los mismos sitios de estudio en el Valle de TehuacánCuicatlán. Ambos estudios tenían por objetivo el analizar la dinámica estacional de la cantidad de materia orgánica y de nutrientes del suelo en IR formadas por Mimosa spp.; como resultado colateral, señalan que el número total de esporas de HMA del suelo tiende a ser mayor durante la estación de lluvias y menor durante la estación de sequía al interior de la IR. Sin embargo, hasta donde sabemos, el trabajo que estamos presentando ahora es el primero en intentar mostrar el efecto de la heterogeneidad espacial y estacional del suelo al interior de las IR formadas por Mimosa spp. y en AA, sobre la distribución y abundancia de esporas de HMA.

Aunque, los resultados muestran un funcionamiento ecológico similar de las distintas especies de Mimosa (IR) como reservorios de esporas de HMA, en el Valle de TehuacánCuicatlán, las diferencias puntuales encontradas en la distribución y abundancia de las esporas de HMA, nos llevan a considerar la necesidad de una serie de estudios mucho más específicos a nivel del hábitat como de la biología propia de las especies de Mimosa formadoras de IR y de las especies vegetales establecidas en la IR.

De acuerdo con algunos investigadores, las grandes variaciones en el número de esporas de HMA pueden estar ligadas a patrones estacionales de la esporulación, la cual puede variar de acuerdo a la especie de HMA o de la planta (Mosse y Bowen 1968, Dhillion y Anderson 1993, Dhillion et al. 1994, Jacobson 1997). De la misma manera, algunos estudios 


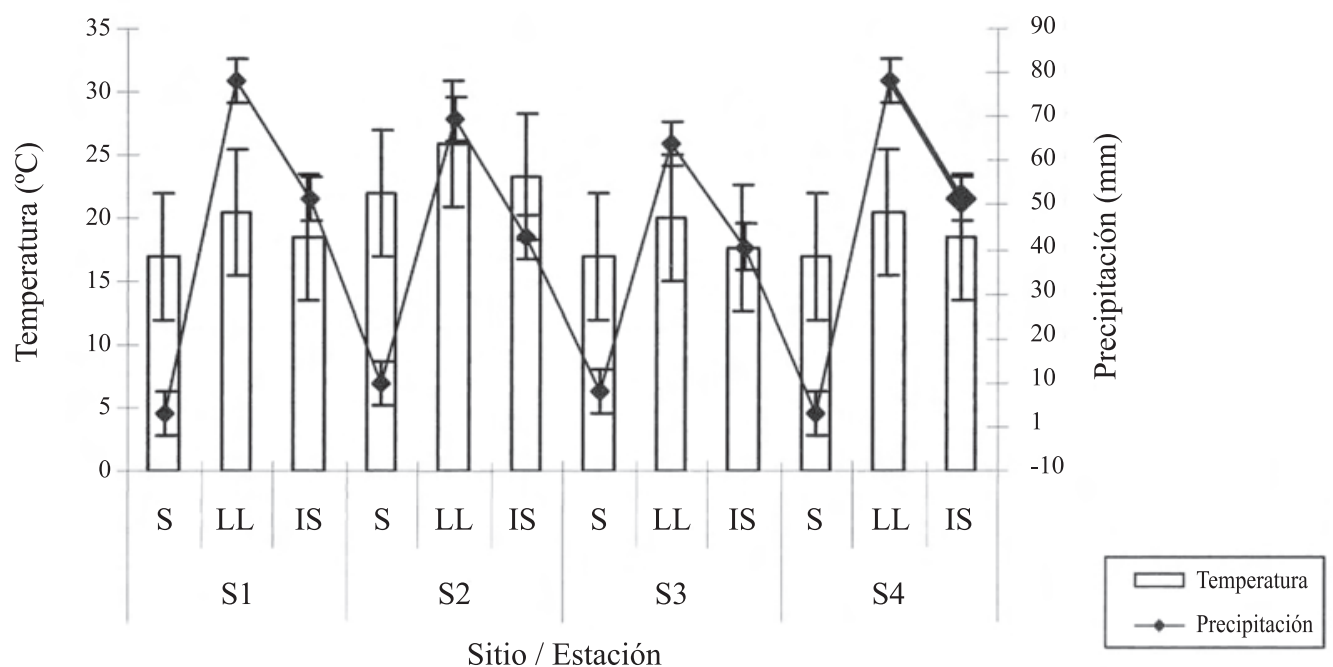

Fig. 2. Promedio de temperatura $\left({ }^{\circ} \mathrm{C}\right)$ y precipitación $(\mathrm{mm})$ mensuales correspondientes a los cuatro sitios de estudio durante las estaciones del año analizadas, en el Valle de Tehuacán-Cuicatlán, Puebla, México. Los promedios de temperatura y precipitación media mensual se obtuvieron con los datos promedio del mes de recolecta (marzo, julio y noviembre) y los dos meses anteriores: lluvias (mayo, junio y julio), inicio sequía (septiembre, octubre y noviembre) y sequía (enero, febrero y marzo). Los datos de temperatura y de precipitación fueron tomados de las estaciones climatológicas más cercanas a los sitios de estudio. Los valores son el promedio de las estaciones climatológicas de Tehuacán: 19 años (S1 y S4); Caltepec: 29 años (S3) y Coxcatlán: 38 años (S2). Sitio 1: S1; Sitio 2: S2; Sitio 3: S3; Sitio 4: S4. Estaciones: Sequía: S; Lluvia: LL; Inicio de Sequía: IS.

Fig. 2. Monthly mean temperature $\left({ }^{\circ} \mathrm{C}\right)$ and precipitation $(\mathrm{mm})$, data correspond to the four study sites during the seasons of the year analyzed within the Tehuacán-Cuicatlán Valley, Puebla, Mexico. Monthly mean temperature and precipitation were obtained by the average of the data of the month of soil collection (March, July and November) plus the two former months: wet season (May, June and July), start of dry season (September, October and November) and dry season (January, February and March). The temperature and precipitation data were taken from the nearer climatological stations to the study sites. The values are the mean of the climatological stations of Tehuacán: 19 years (S1 and S4); Caltepec: 29 years (S3) and Coxcatlán: 38 years (S2). Site 1:S1; Site 2:S2; Site 3:S3; and Site 4: S4. Seasons: Dry: S; Wet: LL; Start of dry: IS.

han determinado que la distribución, actividad y supervivencia de los HMA puede ser influenciada por varios factores y procesos físicos, químicos y biológicos del suelo como son la fertilidad y la humedad (Anderson et al. 1984), la compactación (Nadian et al. 1997, 1998), la profundidad (Virginia et al. 1986), el movimiento físico y la saturación de agua (Cooke et al. 1993, Miller 2000), el pH (Green et al. 1976) y la topografía; también influyen la frecuencia de incendios y los disturbios ambientales (Gibson y Hetrick 1988), la temperatura (Koske 1987), la intensidad de luz, la altitud y la latitud (Allen et al. 1995), la susceptibilidad fenológica de las plantas y las variaciones fenológicas de los HMA (Sylvia et al. 1993); además de la actividad propia de la micro y mesofauna del suelo (Hayman 1982).

Asimismo, McGee (1989) y Allen (1991) señalan que las diferencias en el número de esporas de HMA del suelo también podrían estar relacionadas con diferentes estrategias de supervivencia de las especies de HMA al habitar en un ecosistema determinado; es decir, el ciclo de vida de los HMA presenta una alta adaptación al ambiente que los rodea, sobre 
todo durante la etapa de formación de esporas (Camargo-Ricalde 2002) y su habilidad competitiva puede ser afectada por diversos factores, tales como la fenología propia de cada especie de HMA, la frecuencia de inóculo y el tiempo diferencial de incubación, la distribución espacial de los propágulos que interactúan (Wilson 1984, Koske 1987), el rasgo genético específico del HMA (Sanders et al. 1996), y la presencia de ectomicorrizas (Moyersoen et al. 1998); así como por las especies de las plantas hospederas $\mathrm{y}$ las condiciones ambientales particulares.

Otros factores que pueden estar relacionados con estas diferencias en número y distribución de esporas de HMA son: a) La morfología y los exudados específicos de la raíz de las especies de Mimosa, así como la presencia de nódulos fijadores de nitrógeno; sin embargo, no hay estudios al respecto, b) La comunidad microbiana que se desarrolla en el suelo de cada sitio (Garbaye 1991, Dhillion y Zak 1993, Whitford 1996), en el caso particular del Valle de Tehuacán-Cuicatlán (en general y en las IR estudiadas), a la fecha, se desconoce; asimismo, es muy escasa la información relacionada con la dinámica de poblaciones (depredación, dispersión y germinación) de las esporas de HMA del suelo, c) La estabilidad, estructura y contenido de nutrientes del suelo de las IR, en comparación con las AA; de manera general, existen diferencias en la cantidad de materia orgánica y nutrientes entre los cuatro sitios estudiados en el Valle (IR > AA) (CamargoRicalde et al. 2002), d) La microtopografía del suelo (Gibson y Hetrick 1988), e) El grado e intensidad de disturbios ambientales (Allen 1991, Dhillion 1999, Carrillo-García et al. 1999); en el Valle, el sobrepastoreo y la deforestación son las principales causas de disturbio ambiental (Zavala-Hurtado y Hernández-Cárdenas 1998), afectando directamente los cuatro sitios de estudio, f) El tipo de comunidad vegetal establecida en cada sitio, g) La comunidad de arbustos (principalmente leguminosas), herbáceas anuales, bianuales y perennes (principalmente cactáceas, agaváceas, asteráceas y gramíneas) que se establecen en las IR, así como la morfología y exudados específicos de sus raíces, que actualmente están en estudio, y h) El área de la IR, tomada a partir del tamaño de la copa del arbusto o árbol.

Por otra parte, existe controversia al relacionar el número de esporas de HMA y la colonización micorrízica de la raíz (Hayman y Stovold 1979); es decir, el número de esporas de HMA del suelo, no necesariamente está reflejando la capacidad de colonización de estos hongos. En el caso del Valle de TehuacánCuicatlán, Camargo-Ricalde et al. (2003), demostraron que 45 de 50 especies de plantas vasculares estudiadas, formaban micorrizas; aunque, el porcentaje de colonización de las raíces fue bajo, señalan la importancia de hacer observaciones en períodos anuales, dado que la asociación micorrízica puede variar a través del tiempo y del espacio (Dhillion y Zak 1993). En forma adicional, Hayman (1982) ya había demostrado que las esporas de HMA pueden sobrevivir por un año y que puede haber fluctuaciones en la densidad de esporas debidas a las especies de HMA involucrados, y al estado fenológico y especie de la planta hospedera.

En los ecosistemas áridos y semiáridos el estrés primario impuesto sobre la vegetación es la alta temperatura y la falta de humedad óptima durante la mayor parte del año, y la baja disponibilidad de nitrógeno del suelo (Dhillion y Zak 1993), por lo que la formación de micorrizas arbusculares puede ser una ventaja para las plantas que habitan en estos ecosistemas, ya que forman parches ricos en nutrientes y agua que favorecen el establecimiento de plantas, mantienen la diversidad vegetal (Hartnett y Wilson 1999, Koide et al. 2000), y el funcionamiento y la regeneración natural de los ecosistemas (Allen y Allen 1986, Whitford 1996).

Las relaciones entre la diversidad de HMA del suelo y la dinámica de las comunidades de plantas y de los procesos funcionales de los ecosistemas, así como los mecanismos evolutivos que generan la diversidad de especies de HMA, siguen siendo poco comprendidos (Zak y Visser 1996). Aunque los HMA son simbiontes obligados de la raíz de ca. $80 \%$ de las plantas terrestres (Pirozynski 1981), se conoce muy poco de su dinámica poblacional; aunque, se 
han establecido algunos supuestos a través del estudio de la distribución natural de sus esporas (Johnson et al. 1992, Harnett y Wilson 1999).

\section{AGRADECIMIENTOS}

A Rosaura Grether, del Depto. de Biología de la UAM-Iztapalapa, por la revisión crítica del manuscrito, y a Andrea G. Hernández Soriano y a Laura Díaz Antaño por su ayuda en la recolecta del material edáfico.

\section{RESUMEN}

Estudios recientes señalan que algunas especies de Mimosa (Leguminosae-Mimosoideae) forman islas de recursos (IR), ricas en materia orgánica y nutrientes del suelo, así como con esporas de hongos micorrizógenos arbusculares (HMA), en el Valle semiárido de Tehuacán-Cuicatlán. La importancia de este hecho radica en que las zonas áridas y semiáridas se caracterizan por la baja fertilidad del suelo y por su escasa precipitación, lo que limita el crecimiento y desarrollo de las especies vegetales, de manera que la presencia de HMA puede ser una ventaja para las plantas desérticas que forman micorrizas. Las fluctuaciones en el número de esporas de HMA del suelo pueden estar relacionadas con factores ambientales, edáficos y estacionales que afectan la esporulación de estos hongos, así como la historia de vida de la planta hospedera. El objetivo de este estudio fue evaluar el impacto de la heterogeneidad espacial (IR vs áreas abiertas, AA) y estacional (estación de lluvias vs inicio de sequía $v s$ sequía) del suelo sobre la distribución y abundancia del número de esporas de HMA, en cuatro sitios de estudio en el Valle de Tehuacán-Cuicatlán. Se registraron esporas de HMA en las 120 muestras de suelo analizadas. Se registraron diferencias significativas al comparar entre el número de esporas de HMA del suelo bajo el dosel de las especies de Mimosa (IR) y el de las AA (IR > AA), así como al comparar entre los tres puntos de muestreo de suelo de las IR (suelo cercano al tronco > suelo debajo de la parte media de la copa > suelo debajo del margen de la copa). Sin embargo, no se registró diferencia significativa entre el número de esporas del suelo cercano al tronco y el del medio, así como entre el suelo del margen y el del AA. Finalmente, se reportó un mayor número de esporas en el suelo durante la estación de lluvias que durante la estación de sequía (lluvias $>$ inicio de sequía > sequía). Por tanto, la distribución de esporas de HMA es afectada por la heterogeneidad espacial y la heterogeneidad estacional del suelo. Este estudio señala la importancia de las IR formadas por Mimosa como reservorios de esporas de HMA y la importancia potencial de estos hongos para la supervivencia y el establecimiento de las plantas en regiones semiáridas. Recientemente, los HMA han sido reconocidos como uno de los factores importantes en la determinación de la diversidad de especies vegetales en ecosistemas áridos y templados.

Palabras clave: esporas de hongos micorrizógenos arbusculares (HMA), heterogeneidad espacial, heterogeneidad estacional, islas de recursos (IR), Mimosa, semiaridez, suelo.

\section{REFERENCIAS}

Allen, M.F. 1991. The ecology of Mycorrhizae. Cambridge University. Nueva York. 184 p.

Allen, E.B. \& M.F. Allen. 1986. Water relations of xeric grasses in the field: Interactions of mycorrhizas and competition. New Phytol. 104: 559-571.

Allen, E.B., M.F. Allen, D.J. Helm, J.M. Trappe, R. Molina \& E. Rincón. 1995. Patterns and regulation of mycorrhizal plant and fungal diversity. Plant Soil 170: 47-62.

Anderson, R.C., A.E. Liberta \& L.A. Dickman. 1984. Interaction of vascular plants and vesicular-arbuscular mycorrhizal fungi across a soil moisture-nutrient gradient. Oecologia 64: 111-117.

Camargo-Ricalde, S.L. 2002. Dispersal, distribution and establishment of arbuscular mycorrhizal fungi: a review. Bol. Soc. Bot. México 71: 33-44.

Camargo-Ricalde, S.L., S.S. Dhillion \& R. Grether. 2002. Community structure of endemic Mimosa species and environmental heterogeneity in a semi-arid Mexican valley. J. Veg. Sci. 13: 697-704.

Camargo-Ricalde, S.L. \& S.S. Dhillion. 2003. Endemic Mimosa species can serve as mycorrhizal "resource islands" within semiarid communities of the TehuacánCuicatlán Valley, Mexico. Mycorrhiza 13: 129-136.

Camargo-Ricalde, S.L., S.S. Dhillion \& C. JiménezGonzález. 2003. Mycorrhizal perennials of the "matorral xerófilo" and the "selva baja caducifolia" communities in the semiarid Tehuacán-Cuicatlán Valley, Mexico. Mycorrhiza 13: 77-83.

Carrillo-García, A., J.L. León de la Luz, Y. Bashan \& G.J. Bethlenfalvay. 1999. Nurse plants, mycorrhizae and plant establishment in a disturbed area of the Sonoran Desert. Rest. Ecol. 7: 321-335.

Cooke, J.C., R.H. Butler \& G. Madole. 1993. Some observations on the vertical distribution of vesicular myorrhizae in roots of salt marsh grasses growing in saturated soil. Mycologia 85: 574-550. 
Dhillion, S.S. \& R.C. Anderson. 1993. Seasonal dynamics of dominant species of arbuscular mycorrhizae in burned and unburned sand prairies. Can. J. Bot. 71: $1625-1630$

Dhillion, S.S., M.A. McGinley, C.F. Friese \& J.C. Zak. 1994. Construction of sand shinnery oak communities of the Llano Estacado: animal disturbances, plant community structure and restoration. Rest. Ecol. 2: $51-60$.

Dhillion, S.S. \& J.C. Zak.1993. Microbial dynamics in arid ecosystems: desertification and the potential role of Mycorrhizas. Rev. Chil. Hist. Nat. 66: 253-270.

Dhillion, S.S. 1999. Environmental heterogeneity, animal disturbances, microsite characteristics, and seedling establishment in Quercus havardii community. Rest. Ecol. 7: 399-406.

Garbaye, J. 1991. Biological interactions in the mycorrhizosphere. Experentia 47: 370-375.

Gerdemann, J.W. \& T.H. Nicolson. 1963. Spores of mycorrhizal Endogone species extracted from soil by wet sieving and decanting. Trans. Br. Mycol. Soc. 46: 235-244.

Gibson, A. \& B.A.D. Hetrick. 1988. Topographic and fire effects on the composition and abundance of VAMycorrhizal fungi in tallgrass prairie. Mycologia 80: 433-441.

Green, N.E., S.O. Graham \& N.C. Schenk. 1976. The influence of $\mathrm{pH}$ on the germination of vesicular-arbuscular mycorrhizal spores. Mycologia 68: 929-934.

Hartnett, D.C. \& T.H. Wilson. 1999. Mycorrhizae influence plant community structure and diversity in tallgrass prairie. Ecology 80: 1187-1195.

Hayman, D.S. 1982. Influence of soils and fertility on activity and survival of vesicular-arbuscular mycorrhizal fungi. Phytopathology 72: 1119-1125.

Hayman, D.S. \& G.E. Stovold. 1979. Spore populations and infectivity of vesicular arbuscular mycorrhizal fungi in New South Wales. Aust. J. Bot. 27: 227-233.

Jacobson, K.M. 1997. Moisture and substrate stability determine VA-mycorrhizal fungal community distribution and structure in arid grasslands. J. Arid Environ. 35: 59-75

Johnson, N.C., D. Tilman \& D. Wedin. 1992. Plant and soil controls on mycorrhizal fungal communities. Ecology 73: 2034-2042.

Koide, A.G., M.D. Goff \& I.A. Dickie. 2000. Component growth efficiencies of mycorrhizal and nonmycorrhizal plants. New Phytol. 148: 163-168.
Koske, R.E. 1987. Distribution of VA mycorrhizal fungi along a latitudinal temperature gradient. Mycologia 79: 55-68.

McGee, P. 1989. Variation in propagule numbers of vesicular-arbuscular mycorrhizal fungi in a semi-arid soil. Mycol. Res. 92: 28-33.

Miller, S.P. 2000. Arbuscular mycorrhizal colonization of semi-aquatic grasses along a wide hydrologic gradient. New Phytol. 145: 145-155.

Mosse, B. \& G.D. Bowen. 1968. The distribution of Endogone spores in some Australian and New Zeland soils, and in an experimental field soil at Rothamsted. Trans. Br. Mycol. Soc. 51: 485-492.

Moyersoen, B., A.H. Fitter \& I.J. Alexander. 1998. Spatial distribution of ectomycorrhizas and arbuscular mycorrhizas in Korup National Park rain forest, Cameroon, in relation to edaphic parameters. New Phytol. 139: 311-320.

Nadian, H., S.E. Smith, A.M. Alston \& R.S. Murray. 1997. Effects of soil compaction on plant growth, phosphorus uptake and morphological characteristics of vesicular-arbuscular mycorrhizal colonization of Trifolium subterraneum. New Phytol. 135: 303-311.

Nadian, H., S.E. Smith, A.M. Alston, R.S. Murray \& B.D. Siebert. 1998. Effects of soil compaction on phosphorus uptake and growth of Trifolium subterraneum colonized by four species of vesicual-arbuscular mycorrhizal fungi. New Phytol. 139: 155-165.

Newsham, K.K., A.R. Watkinson \& A.H. Fitter. 1995. Rhizosphere and root infecting fungi and the design of ecological field experiments. Oecologia 102: 230-237.

Pirozynski, K.A. 1981. Interactions between fungi and plants through the ages. Can. J. Bot. 59: 1824-1827.

Sanders, I.R., J. Clapp \& A. Wiemken. 1996. The genetic diversity of arbuscular mycorrhizal fungi in natural ecosystems -a key to understanding the ecology and functioning of the mycorrhizal symbiosis. New Phytol. 133: 123-134.

Schenk, N.C. \& Y. Perez. 1990. Manual of the identification of VA mycorrhizal fungi. Synergistic, Florida. EUA.

Streitwolf-Engel, R., T. Boller, A. Wiemken \& I.R. Sanders. 1997. Clonal growth traits of two Prunella species are determined by co-occurring arbuscular mycorrhizal fungi from a calcareous grassland. J. Ecol. 85: 181-191.

Sylvia, D.M., D.O. Wilson, J.H. Graham, J.J. Madox, P. Millner, J.B. Morton, H.D. Skipper, S.F. Wright \& A.G. Jarstfer. 1993. Evaluation of vesicular-arbuscular 
mycorrhizal fungi in diverse plants and soil. Soil Biol. Biochem. 25: 705-713.

Tarafdar, J.C. \& Praveen-Kumar. 1996. The role of vesicular-arbuscular mycorrhizal fungi on crop, tree and grasses grown in an arid environment. J. Arid Environ. 34: 197-203.

Titus, J.H. \& R. del Moral. 1998a. Vesicular-arbuscular mycorrhizae influence Mount St. Helens pioneer species in greenhouse experiments. Oikos 81: 495-510.

Titus, J.H. \& R. del Moral. 1998b. The role of micorrhizal fungi and microsites in primary succession on Mount St. Helens. Amer. J. Bot. 85: 370-375.

Turner, S. \& C.F. Friese. 1998. Plant-mycorrhizal community dynamics associated with a moisture gradient with a rehabilitated prairie fen. Rest. Ecol. 6: 44-51.

Van der Heijden, M.G.A., J.N. Klironomos, M. Ursic, P. Moutoglis, R. Streitwolf-Engel, T Boller, A. Wiemken \& I.R. Sanders. 1998a. Mycorrhizal fungal diversity determines plant biodiversity, ecosystem variability, and productivity. Nature 396: 69-72.
Van der Heijden, M.G.A., T. Boller, A. Wiemken \& I.R. Sanders. 1998b. Different arbuscular mycorrhizal fungal species are potential determinants of plant community structure. Ecology 79: 2082-2091.

Virginia, R.A., M.B. Jenkins \& W.M. Jarrel. 1986. Depth of root symbiont ocurrence in soil. Biol. Fert. Soil 2: $127-130$.

Whitford, W.G. 1996. The importance of the biodiversity of soil biota in arid ecosystems. Biodivers. Conserv. 5: 185-195.

Wilson, J.M. 1984. Competition for infection between vesicular-arbuscular mycorrhizal fungi. New Phytol. 97: 427-482.

Zak, J.C \& S. Visser. 1996. An appraisal of soil fungal biodiversity: the crossroads between taxonomic and functional biodiversity. Biodivers. Conserv. 5: 169-183.

Zavala-Hurtado, J.A. \& G. Hernández-Cárdenas. 1998. Estudio de caracterización y diagnóstico del área propuesta como Reserva de la Biósfera Tehuacán-Cuicatlán. UniversidadAutónoma Metropolitana-Instituto Nacional de Ecología (INE), SEMARNAP, México. 